\title{
Requirements for AO-ELT operation and ELT site monitor
}

\author{
Andrei Tokovinin ${ }^{1, \text { a }}$ \\ Cerro Tololo Inter-American Observatory. Casilla 603, La Serena, Chile
}

\begin{abstract}
Performance of AO systems on ELTs depends not just on seeing; other parameters such as strength of high-altitude turbulence are more relevant for the laser tomography. Nights good for some instruments are not so good for others, so there is a clear advantage to go from the simple queue scheduling on seeing to a more complex strategy of selecting in a multi-parameter space. The need to know those atmospheric parameters in real time drives the requirements to the ELT site monitors and their data. It is suggested to measure atmospheric parameters and internal seeing along the line of sight by an internal seeing monitor, complementing the external site monitor.
\end{abstract}

\section{Standard and advanced queue scheduling}

Extremely large telescopes (ELTs) will work with adaptive-optics (AO) most of the time. The performance of AO and, consequently, the science output of ELTs, strongly depend on the atmospheric conditions. Operation of ELTs in queue-scheduled (QS) mode, when observations are done under optimum, rather than random, conditions, is an obvious choice. Modern large telescope already work in the QS mode driven by the seeing measured by site monitors.

The QS on seeing will not be the best choice for ELTs, however. Their AO systems will use laser guide stars (LGSs) and tomography and their performance will mostly depend on the turbulent conditions in the high atmosphere. Therefore, a better choice will be to schedule observations on relevant atmospheric parameters related to the quality of $\mathrm{AO}$ science. Those parameters will differ, depending on the type of AO. Let us call this strategy advanced queue scheduling (AQS) to distinguish it from the standard queue scheduling (SQS) on seeing and the classical scheduling (CS) when nights are assigned in advance without regard to atmospheric conditions.

Considering that ELTs and their instruments represent large investment, even a modest gain in productivity achieved by changing the strategy from SQS to AQS is worth the effort. In this contribution, SQS and AQS are compared on two examples using real atmospheric data. Then we detail the requirements to the ELT site monitors and their data products as needed for the AQS.

\section{Atmospheric parameters relevant for AO}

Performance of ELTs depends on different atmospheric parameters (Table 1). Consult [1] for the definitions of those parameters. In the case of seeing-limited observations without $\mathrm{AO}$, the delivered image quality (DIQ) is relevant. It depends on the total seeing $\varepsilon_{0}$ (or, equivalently, on the Fried parameter $\left.r_{0}=0.98 \lambda / \varepsilon_{0}\right)$ and effective turbulence outer scale $L_{0}$. However, the DIQ is also affected by the internal seeing in the ELT and by the quality of its optics.

The DIQ will be improved over a moderately large field by partially compensating low turbulence with Ground-Layer AO (GLAO). The GLAO performance depends on the remaining (un-compensated) seeing in the free atmosphere $\varepsilon_{\text {free }}$ and on the thickness of the near-ground turbulence. Real-time knowledge of the turbulence profile (TP) $C_{n}^{2}(h)$ is needed for GLAO operation. The resolution of the TP has to be high near the ground, but only a coarse TP in the high atmosphere is sufficient.

Most often, AO at ELTs will use several LGSs to reconstruct 3-dimensional instantaneous distortions by means of laser tomography. Such LTAO is necessary because with a single LGS, the cone

\footnotetext{
a e-mail: atokovinin@ctio.noao.edu
}

This is an Open Access article distributed under the terms of the Creative Commons Attribution-Noncommercial License, which permits unrestricted use, distribution, and reproduction in any noncommercial medium, provided the original work is properly cited. 
effect on large ELT apertures becomes prohibitively strong. The information derived from LTAO will serve to compensate turbulence in the direction of the science object, for several selected objects, or over some field (using multi-conjugate AO - MCAO).

The performance of LTAO in general (and MCAO in particular) depends on the TP $C_{n}^{2}(h)$ and on the system parameters in a complicated way. Situations when turbulence is concentrated mostly in few strong layers are more favorable than a continuous TP. A notion of the effective thickness of the atmosphere $\delta_{K}$ has been introduced in [2]. The maximum angular radius of the LGS constellation needed for tomography is estimated as $\gamma_{K}=r_{0} / \delta_{K}$, depending also on the number $K$ of LGSs and their configuration. Actual TPs are such that $\delta_{5}$ is rarely less than $0.5 \mathrm{~km}$. Considering a typical seeing of $r_{0}=0.9 \mathrm{~m}$ (at $2.2 \mu \mathrm{m}$ imaging wavelength), the diameter of the 5-LGS constellation for LTAO (and the diameter of the science field) will be $2 \gamma_{K} \sim 12^{\prime}$ or less. This is $\sim 20$ times larger than the typical isoplanatic angle $\theta_{0} \sim 15^{\prime \prime}$. It is argued in [2] that LTAO can measure TP with a vertical resolution of the order of $\delta_{K}$. Conversely, this parameter indicates the minimum TP resolution needed for predicting LTAO performance.

Table 1. Atmospheric parameters for advanced ELT queue-scheduling

\begin{tabular}{ll}
\hline Operational mode & Atmospheric parameters \\
\hline Seeing-limited & Seeing $\varepsilon_{0}, L_{0}$, DIQ \\
GLAO & Turbulence profile $C_{n}^{2}(h)$ \\
LTAO, MCAO & TP $C_{n}^{2}(h)$, LTAO angle $\gamma_{K}$, time constant $\tau_{0}$ \\
Extreme AO & Seeing $\varepsilon_{0}$, time constant $\tau_{0}$ \\
\hline
\end{tabular}

Finally, extreme AO (ExAO) uses natural guide stars (no cone effect), but aims at very high degree of compensation. In this mode, the relevant parameters are the integrated seeing and the AO time constant $\tau_{0}$.

\section{Examples of advanced queue scheduling}

We try to compare quantitatively three types of scheduling: CS, SQS, and AQS. The TPs measured at Cerro Tololo with the robotic MASS-DIMM site monitor [3] over the period of 4.8 years are used as input data. Those TPs have low vertical resolution (logarithmic grid of 6 layers from $0.5 \mathrm{~km}$ to $16 \mathrm{~km}$ and the ground layer) and time resolution of $1 \mathrm{~min}$. The AO time constant $\tau_{0}$ was estimated simultaneously by the same monitor. The data were kindly prepared by S. Els. To make the data volume manageable, we used only every 50-th TP, or 8517 TPs out of 433161, which is equivalent to sampling randomly the conditions every hour.

We consider as a test case 1 an AO system with a single sodium LGS at $90 \mathrm{~km}$ altitude working at the 8-m telescope such as Gemini or VLT. The tip-tilt NGS is at $30^{\prime \prime}$ from the science object. To highlight LGS-specific errors (cone effect and tilt anisoplanatism), the system has a very high compensation order (240 modes) and the servo lag error is neglected. For each TP, the PSF on-axis and at $20^{\prime \prime}$ offset in the $\mathrm{H}$ band $(\lambda=1.65 \mu \mathrm{m})$ is calculated by the fast Fourier method outlined in [4]. Such AO system would have on-axis Strehl ratio of 0.95 under median condition if used with NGS, but the actual median Strehl is 0.25 .

As case 2, a 42-m telescope with a similarly high-order AO and a tip-tilt NGS at 30" is modeled. With a single sodium LGS, the cone effect is so important that even in the K-band $(\lambda=2.2 \mu \mathrm{m})$ the median Strehl on-axis is 0.11. Although the use of LTAO will be typical for ELTs, this simple test case with a single LGS is chosen to highlight the role of high-altitude turbulence, also relevant for LTAO.

Figure 1 plots the on-axis Strehl ratio for the 8-m telescope as a function of seeing and isoplanatic angle. The performance is correlated with the seeing only poorly because it is determined mostly by the high-altitude turbulence, whereas the seeing is mostly produced in the low atmospheric layers. On the other hand, the relation between Strehl and isoplanatic angle $\theta_{0}$ is nearly perfect. Partially, this 

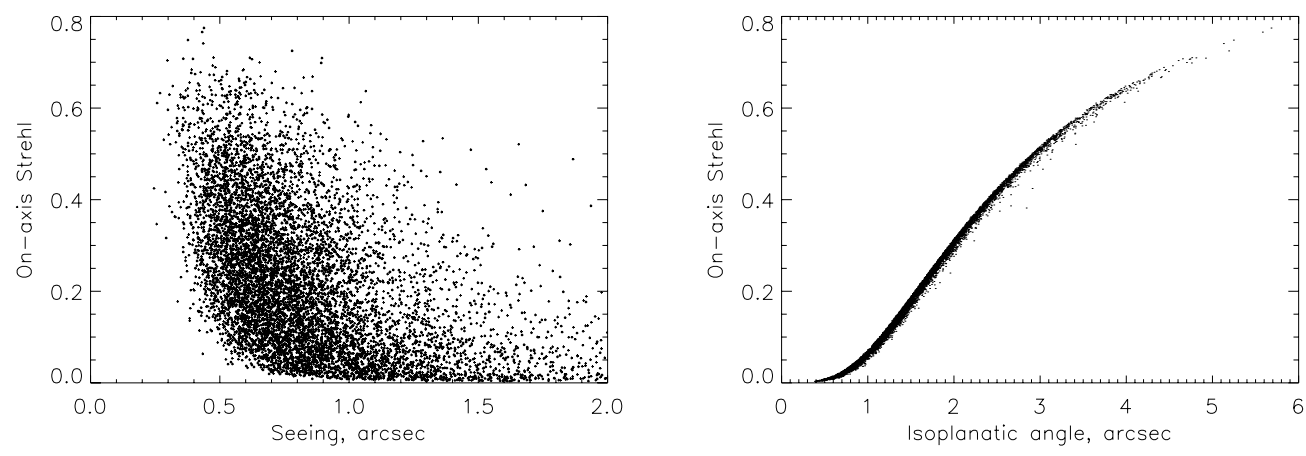

Fig. 1. On-axis Strehl ratio for an 8-m telescope (case 1) as a function of seeing (left) and isoplanatic angle (right).
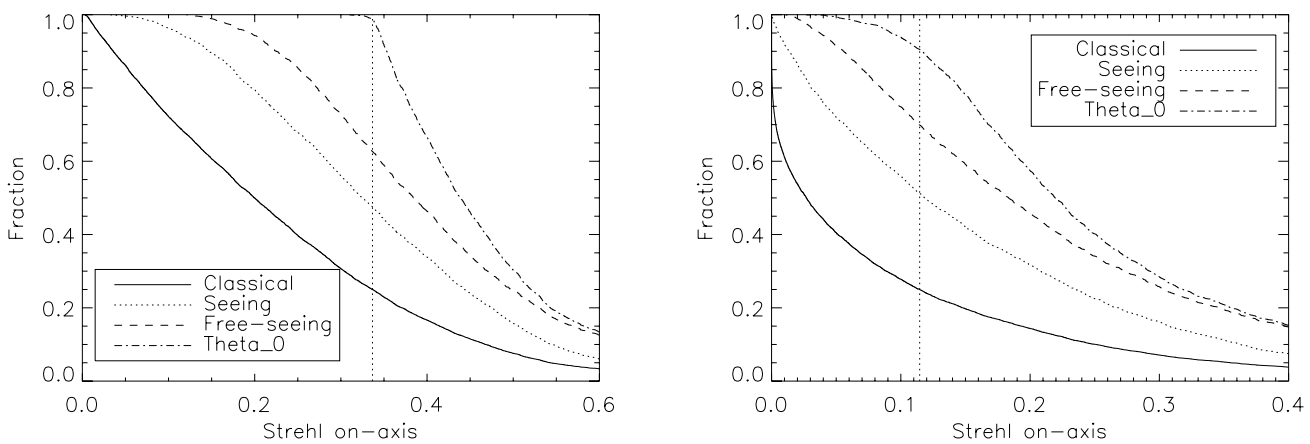

Fig. 2. The full lines show the probability of obtaining a Strehl ratio better than a given value, with vertical lines marking the best quartile. The dotted, dashed, and dash-dotted lines show the Strehl distributions for the best $25 \%$ of nights selected according to $\varepsilon_{0}, \varepsilon_{\text {free }}$, and $\theta_{0}$, respectively. The left plot refers to the case 1 (8-m telescope, H-band), the right plot to the case 2 (ELT, K-band), both working at Cerro Tololo.

correlation is an artifact of a low TP resolution. Anyway, in this case, using $\theta_{0}$ as a queue-scheduling parameter will give better results than using seeing.

The overall distribution of Strehl ratios $S$ for the case 1 is plotted in Fig. 2 (left) in full line. This distribution corresponds to the performance of an AO system with CS (no relation to conditions). For the sake of argument, suppose that the science program requires the best-quartile AO performance, $S>0.33$. Only one night out of 4 will produce useful data.

Suppose now that the same AO program gets best $25 \%$ of nights selected by some atmospheric parameter. If the selection is done on seeing $\varepsilon_{0}$ (SQS), successful observations will be obtained with the probability of $50 \%$. A somewhat better result will be achieved if we use free-atmosphere seeing $\varepsilon_{\text {free }}$ (excluding turbulence below $0.5 \mathrm{~km}$ ) as a scheduling criterion. However, nearly guaranteed performance is delivered if AQS is based on the isoplanatic angle $\theta_{0}$. Therefore, there remains no doubt that the relevant atmospheric parameter in this case will be the isoplanatic angle, rather than seeing, and that AQS on this parameter can improve the science return from the AO system by as much as two times.

In the case 2 (ELT), $\theta_{0}$ is also correlated with the Strehls, but not so tightly. The conclusion on the relative merits of CS, SQS and AQS remains the same (Fig. 2, right).

The above two examples do not mean that $\theta_{0}$ should be used for AQS in all cases. They are provided as an illustration of a study to be done for different $\mathrm{AO}$ systems. The problem of defining relevant parameters is complicated and does not have a unique answer. For each instrument, the statistics of its 
performance metric for a typical use case can be determined, and then the combination of atmospheric parameters which shows the best correlation with the metric will be identified as the relevant parameter for this specific case.

We end up with a multitude of atmospheric parameters relevant for ELT instruments or their operational modes. Even if the number of these parameters is restricted to a minimum, will it be practical to schedule ELT operation in such a complex way? We believe, yes. Given the adequate input information from the site monitor, an optimization software will indicate the best (and second-best) choice of the instrument and program to be executed at any given time. Multi-dimensional parameter space for queue scheduling is advantageous because bad conditions for one instrument can be acceptable or good for another. For example, a strong near-ground turbulence, detrimental for ExAO, will be compensated well by the GLAO. The loss of valuable ELT time due to inadequate AO performance will be less with the AQS then with the SQS.

Practically, we may find out that the transition from SQS at AQS can be smooth. A multitude of relevant parameters can be replaced by a small number of "proxies" which are not as good, but still show a better correlation with AO performance than just seeing. Most likely, the next-important relevant parameter will be related to the strength and speed of turbulence in the high atmosphere. As this turbulence is often generated by the jet stream, which is itself predictable, the AQS will benefit from the look-ahead knowledge of the atmosphere. Prognostic of the jet stream is more reliable than seeing predictions!

\section{Requirements for ELT site monitors}

Wave-front distortions that affect ELT and are corrected by AO come both from the atmosphere and from inside the telescope - dome and mirror seeing, aberrations induced by gravity and wind loads, vibrations. Some of those internal disturbances are quite fast, blurring the borderline between active and adaptive optics. Internal distortions are already important at the current large telescopes, they will be even more severe at ELTs with their long light path inside dome and larger, less stiff structures. Obviously, we need not one but two site monitors, external and internal - ESM and ISM.

All atmospheric parameters are defined in the framework of the stationary-random-process theory. The actual turbulence is not stationary in space and time, it is as "patchy" as clouds. Seeing and other parameters depend on the viewing direction and change with time. Even if atmospheric parameters were measured accurately by an ESM, they would not be quite relevant to the actual wave-front seen through the ELT. Accurate measurements are illusory anyway because by the time we accumulate enough data to average out random fluctuations the conditions change! So, we should not require high accuracy from an ESM and should try instead to supplement it with measurements obtained through the ELT itself with the ISM, concurrently with the science data. The ISM data will probably come too late to drive the AQS decisions, but they will be a valuable complement to the science data, e.g. for the PSF reconstruction.

An obvious requirement to the ESM is $100 \%$ coverage of observing (clear) night time and the real-time availability of its data. The data products must include such global atmospheric parameters as seeing (at the top of the dome level), isoplanatic angle, and AO time constant. Additionally, realtime measurements of the turbulence profile and outer scale may be required. If the vertical resolution of $C_{n}^{2}(h)$ profiles is driven by the laser tomography, it should be better than $0.5 \mathrm{~km}$. Atmospheric parameters unrelated to turbulence (extinction, sodium profile) may be needed as well.

The best location for an ESM appears to be on top of the ELT dome. Compensating dome rotation to keep the pointing is much easier than extrapolating data from an instrument located in a small tower to the height of 50-100 m. The type of the instrument to be used as ESM will be defined from a compromise between requirements and cost. For example, the simple MASS-DIMM instrument [3] provides all integrated parameters, but the resolution of TP is too low for LTAO, while the estimates of $\tau_{0}$ from MASS-DIMM are only approximate. The AO time constant can be measured accurately by recording rapid fluctuations of defocus with a specially designed instrument like FADE [5] or with a fast low-order Shack-Hartmann sensor. As such sensor is already implemented in SLODAR [6], this instrument can be a good candidate for an ESM. It may well be that none of the existing instruments satisfies all the requirements, calling for a new development. 


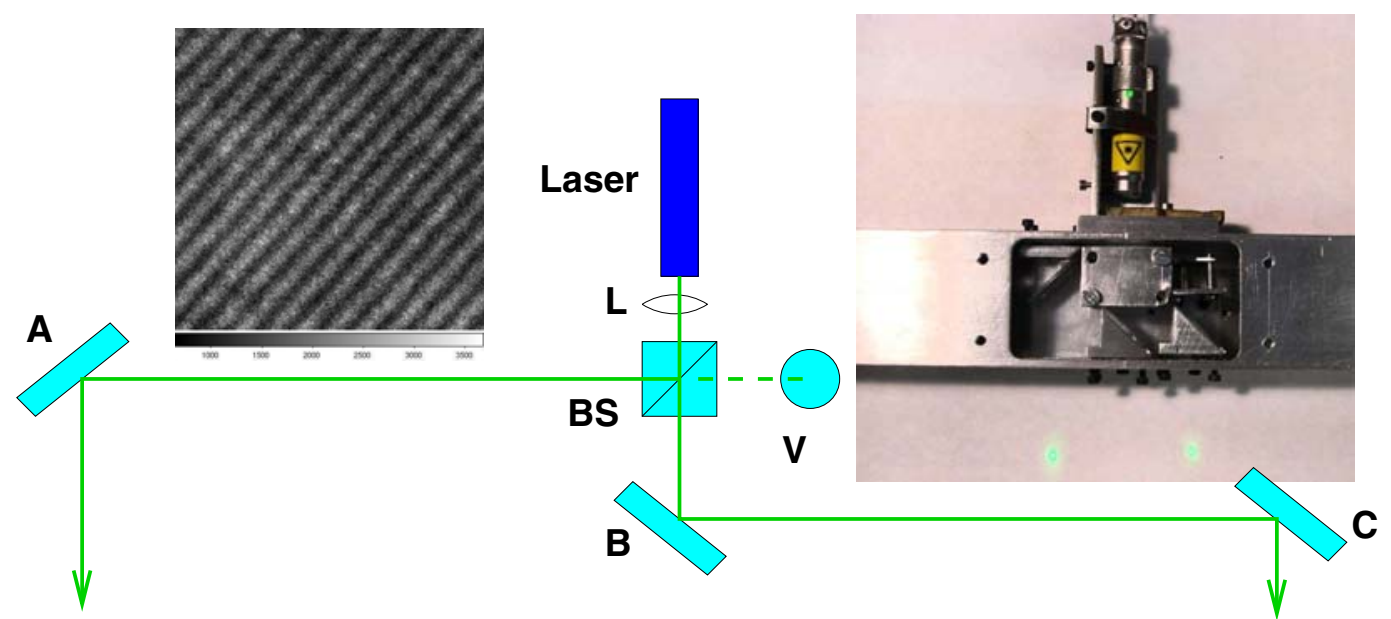

Fig. 3. Schematics of the laser interferometer used at SOAR. The inserts show the fringes (left) and the picture of the central part (right). The baseline is $\mathrm{AC}=0.5 \mathrm{~m}$, wavelength $532 \mathrm{~nm}$.

The internal site monitor, ISM, should sense the turbulence on the light path inside the dome and measure the integrated atmospheric parameters on the line of sight. Seeing can be estimated from the width of the spots in an active-optics Shack-Hartmann sensor (e.g. [7]). On the other hand, any working AO system provides rich data suitable for estimating the seeing and time constant. Methods have been developed to get such estimates [8]. However, atmospheric characterization from AO data is usually of low priority, instrumental biases are not always removed and, as a result, the data on atmospheric parameters supplied routinely by an AO system such as NAOS are not reliable without additional "cleaning". This situation may change if AO development teams are given a clear mandate to supplement each observation with reliable estimates of atmospheric parameters.

Any working LTAO system provides, by definition, data on turbulence profile along the line of sight. Methods to extract such data can vary from a simple SLODAR-like approach to something more elaborate (e.g. [2]). Again, dedicated effort will be needed to ensure that such data are reliable and unbiased, but, on the other hand, they will be much more relevant than a TP supplied by an ESM looking in some other direction. The resolution of the TP derived from LTAO will be matched to the system parameters, alleviating the requirements on the ESM. In this case, scheduling of LTAO operation will be driven by some proxy parameters such as $\theta_{0}$ or turbulence intensity in the high atmosphere, while the actual performance will be known only when the instrument is already working.

Internal seeing in an ELT can be measured by shining diagnostic laser beams through the optics. This extra light can be made inoffensive to the science operation by directing it off-axis and using a different wavelength. For example, we can implement the DIMM method by putting two 5-cm collimators with laser diodes on a spider of the ELT, directing those two beams into the primary mirror at some angle, and detecting the two spots with a small off-axis camera near the focus. The differential and absolute motion of the spots will give a crude idea about internal seeing parameters $\left(r_{0}, L_{0}\right)$, as well as about vibrations of the optics. Such data will be useful for optimizing air flow inside the dome.

Considering that internal turbulence is likely non-Kolmogorov, we may replace two collimators with multiple laser beams to measure the phase structure function. A two-beam interferometer was attached to the spider of the 4-m SOAR telescope in April 2009 for the purpose of pixel-scale calibration, projecting fringes with a well-known period to the focal plane (Fig. 3). As a by-product, the fringe motion was measured. It turned out to be quite small, with rms between 33 and 66 mas (during 3 nights with low wind speed). 


\section{Conclusions}

Using AO on ELTs in classically scheduled mode or in queue scheduling driven by the seeing will not be the best approach. Better science yield will be obtained by flexible scheduling based on multiple atmospheric parameters which are more relevant to the performance of different AO modes than the seeing. The need to measure those parameters in real time drives the requirements to the site monitors. A combination of an external monitor of atmospheric parameters with an internal monitor which provides data on turbulence along the line of sight and, additionally, measures internal seeing, will be the optimum choice to ensure the most efficient ELT operation and to accompany its science data with relevant and reliable information on the observing conditions.

I am grateful to S. Els for extracting and calibrating the data of the CTIO site monitor used in this study.

\section{References}

1. Roddier F. 1981, Progres in Optics, 19, 281-376

2. Tokovinin A., Viard E. 2001, JOSA(A), 18, 873-878

3. Kornilov V., Tokovinin A., Shatsky N. et al. 2007, MNRAS, 382, 1268-1278

4. Tokovinin A. 2004, PASP, 116, 941-951

5. Tokovinin A., Kellerer A., Coude Du Foresto V. 2008, A\&A, 477, 671-680

6. Butterley T., Wilson R., Sarazin M. 2006, MNRAS 369, 835

7. Tokovinin A., Sarazin M., Smette A. 2007, MNRAS, 378, 701-708

8. Fusco T., Rousset G., Rabaud D. et al. 2004, J. Opt. A: Pure Appl. Opt. (Paris), 6, 585-596 